patients showed lower score in general health domain than RA patients (age and sex-adjusted scores, $41.4 \pm 1.8$ vs $51.3 \pm 1.8, \mathrm{p}<0.001)$. Moreover, SSc patients reported remarkably lower scores of EQ-5D-3L index scores than all comparative rheumatic disease groups (RA, SLE, SJS) $(p<0.001)$. HRQoL of patients with SSc was associated with HAQ-DI and SSc HAQ-DI.

Abstract AB0753 - Table 1. Comparisons of the SF-36, SF-6D and EQ-5D-3L, adjusted by age and sex in study subjects.

\begin{tabular}{|c|c|c|c|c|c|c|}
\hline & \multicolumn{6}{|c|}{ Greap } \\
\hline & 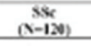 & $\underset{(N-120)}{R A}$ & 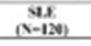 & $\underset{d=13 n}{\mathbf{s}}$ & $\begin{array}{l}\text { Conterol } \\
(\mathrm{N}-6 \mathrm{CO})\end{array}$ & $\operatorname{Ann} a P^{\prime}$ \\
\hline Prignicel fatction & $705(2.21$ & $03(22)$ & 6821221 & $739(22)$ & $32+1100$ & -9.9n \\
\hline Relophysiad & 6780.33 & $05(23)$ & 6580.33 & $68.6(2.4)$ & 3650.13 & a.0n \\
\hline Bediy pain & 61.1223 & $61+(22)$ & $461(22)$ & $63.1(23)$ & $31+4104)$ & a.6n \\
\hline Conmal hadit & $41.4(1.8)^{4}$ & $51.3(1.3)$ & $44:(1.8)$ & ess (1.s) & os.gpasy & ca.en \\
\hline venitity & 9321199 & $518(1.9)$ & $49 \times 119$ & $\cos x(1.9)$ & $646 \tan$ & 9.011 \\
\hline Soxid finction & 1241211 & $n s(2.1)$ & 2461211 & 313211 & 873010 & 2001 \\
\hline Relo-mocional & $68+12.31$ & $n 2033$ & 1291231 & 6062.4 & 89.90 .11 & and \\
\hline Noves: health & $613(1.8)^{7}$ & $7.9(1.3)$ & 4921189 & $6.2(1.3)$ & 132008 & - $4.0 n$ \\
\hline 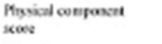 & $44.6(0.8)$ & $253(0.1)$ & $44.8(0.5)$ & $26.9(0.8)$ & $5(1.0(0.4)$ & ca.en \\
\hline Noxal ocepotescessork & $43.010 .9 / 5$ & ผ.9(0.9) & 4661099 & $42(0.9)$ & 50.800 .4 & 9.811 \\
\hline $\mathbb{S}=\mathbb{B}$ & aso(\$.01) & $0.73+20.01)$ & $\operatorname{arz}(0.01)$ & $0.718001)$ & aryons) & 9.911 \\
\hline 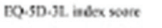 & $0.750 .00 y^{\prime}$ & es1 (a.01) & ase (0.01) & ex (a.01) & a90(001) & 9.011 \\
\hline BQVAS & $6398(155)$ & $6295(1.56)$ & 67.49 (1.55) & $6484(158)$ & $n s s(0.72)$ & 9.91 \\
\hline
\end{tabular}

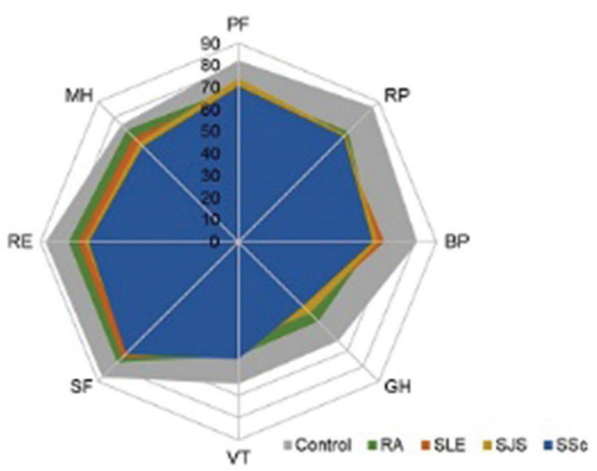

Conclusions: HRQoL of patients with systemic autoimmune diseases is significantly worse and affects all health domains in comparison to healthy controls. Patients with SSc have poorer HRQoL than patients with other rheumatic diseases. Specifically, SSc patients have more impaired mental health than RA patients, and the perception of an individual's general health is also poor compared to RA.

Disclosure of Interest: None declared

DOI: 10.1136/annrheumdis-2018-eular.2264

\section{AB0754 LONG-TERM FOLLOW-UP OF 214 PRIMARY RAYNAUD S PHENOMENON PATIENTS}

F.M. Ortiz Sanjuan, C. Alcañiz Escandell, K.R. Arevalo Ruales, I. Chalmeta Verdejo , M. De la Rubia. Navarro, C. Feced Olmos, J.J. Fragio Gil, R. Gonzalez Mazario, L. Gonzalez Puig, E. Labrador Sánchez, I. Martinez Cordellat, R. Negueroles Albuixech, E. Grau Garcia, J. Ivorra Cortés, J.E. Oller Rodriguez, E. Vicens Bernabeu, C. Najera Herranz, I. Canovas Olmos, J.A. Roman Ivorra. Rheumatology Department, HUP La Fe, Valencia, Spain

Background: Raynaud's phenomenon (RP) is frequently associated with the presence of scleroderma or other connective tissue diseases (CTD). Identify the presence of secondary RP is important to perform an adequate therapeutic management and to achieve the early control of these patients. Nailfold capillaroscopy is safe, economic, and relatively easy to perform and has proven to be useful in identifying patients with secondary RP.

Objectives: To assess a long-term follow-up primary RP patient's series.

Methods: Retrospective observational study of a wide and unselected series of patients diagnosed as primary RP from a single university hospital from January 2012 to August 2017. Patients were classified as primary RP after the presence of CTD at the onset was excluded.
Results: We studied 214 patients (85.98\% were female), with a mean age of 47.6 \pm 16.7 years (range $15-88$ ). After a mean follow-up period of $46.4 \pm 23.3$ months, 8 patients were diagnosed of a CTD (2 Scleroderma/Systemic sclerosis, 3 Systemic Lupus Erythematosus, 1 Rheumatoid arthritis and 2 Sjogren's syndrome). The remaining 206 patients continued classified as primary RP

The main capillaroscopic patterns observed were: Normal $(n=157)$, unspecific $(n=49)$, scleroderma pattern $(n=2)$ and suggestive of other rheumatic diseases pattern $(n=6)$.

All patients who developed a CTD during the follow-up, showed changes in suc cessive nailfold capillaroscopic examinations. 20 of 206 patients who remained primary RP showed minor changes at successive nailfold capillaroscopy. The main capillaroscopic changes detected on this group were: presence of capillary tortuosity $(n=4)$, presence of a decreased capillary density ${ }^{2}$ and the presence of capillary bleeding $(n=16)$.

Antinuclear antibodies (ANA) were positive at the onset in 34 patients and after follow-up in 39 patients.

Conclusions: After a mean follow-up period next to four years, most of our primary RP patients remained free of CTD. A minority of our patients showed changes at nailfold capillaroscopy exam or positivity of ANA.

Disclosure of Interest: None declared

DOI: 10.1136/annrheumdis-2018-eular.6318

\section{AB0755 SKIN SCORE PROGRESSION AFTER DISCONTINUATION OF MYCOPHENOLATE TREATMENT IN SYSTEMIC SCLEROSIS PATIENTS}

F.A. Mendoza ${ }^{1}$, C.P. Lee Ching ${ }^{1}$, S.A. Jimenez ${ }^{2}$. ${ }^{1}$ Scleroderma Center and Division of Rheumatology; ${ }^{2}$ Scleroderma Center and Jefferson Institute of Molecular Medicine, THOMAS JEFFERSON UNIVERSITY, Philadelphia, USA

Background: Rapid progressive skin involvement in diffuse Systemic Sclerosis (rp-dcSSc) is associated with higher mortality and internal organ involvement. Treatment with Mycophenolate Mofetil (MMF) has been shown to halt the progres sion of the disease. However, the optimal duration of therapy is unknown and has not been studied. We follow up a known cohort of rp-dcSSc patients treated with MMF after discontinuation of therapy.

Objectives: To describe the progression of skin involvment in rp-dcSSc patients after discontinuation of MMF

Methods: Twenty-five previously untreated consecutive patients with recentonset $(<24 \mathrm{mo}$ ) rp-dcSSc received MMF as the only disease-modifying therapy. Their Modified Rodnan skin score (mRSS) and Pulmonary function tests were followed after discontinuation of MMF after 2 years of treatment. Patients were followed up every 3-6 months. Therapy was re-initiated if their mRSS increased more than $20 \%$ or worsening respiratory symptoms with progression of restrictive lung disease were reported.

Results: Six patients lost follow up after terminating the open label trial with MMF. From the 19 patients followed up after MMF discontinuation, $26.3 \%{ }^{5}$ required to resume MMF. All these patients required to resume MMF within 6 months after discontinuation. All of them presented increase in mRSS. Two of them (10.5\%) presented respiratory symptoms associated with restriction pattern at PFTs. Skin score returned to baseline in $80 \%$ of the patients after resuming therapy.
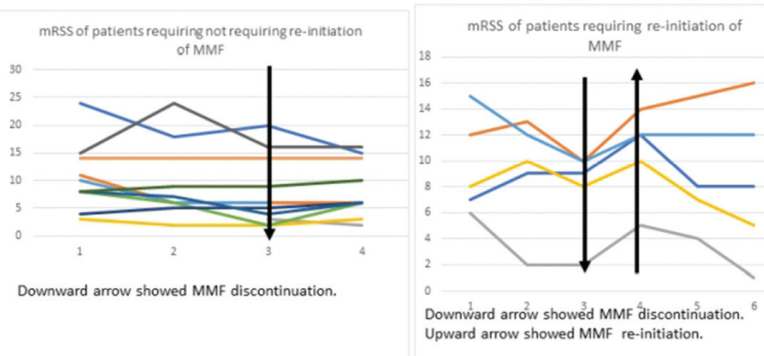

Abstract AB0755 - Figure 1. mRSS after MMF discontinuation

Conclusions: Recurrent skin progression occurs in up to $26.3 \%$ of patients with $\mathrm{rp}$-dcSSc after discontinuation of 2 years of MMF, requiring longer term immunosuppression. In this group, all patients presented active skin disease recurrence within 6 months of treatment discontinuation. Ergo, slow decrease of MMF dose over time and very close follow up is recommended in patients with rp-dcSSc discontinuing MMF even after a prolonged period of time. In addition, these findings support the therapeutic effect of MMF in rp-dcSSc. 